\title{
Effects of addition of rituximab to chemotherapy on central nervous system events in patients with diffuse large B-cell lymphoma
}

\author{
MAN FAI LAW ${ }^{1,2^{*}}$, HAY NUN CHAN ${ }^{1}$, HO KEI LAI ${ }^{1}$, \\ CHUNG YIN HA ${ }^{1}$, CELIA NG ${ }^{1}$, YIU MING YEUNG ${ }^{1}$ and SZE FAI YIP ${ }^{1 *}$ \\ ${ }^{1}$ Department of Medicine, Tuen Mun Hospital; ${ }^{2}$ Department of Medicine and Therapeutics, \\ Prince of Wales Hospital, Hong Kong SAR 999077, P.R. China
}

Received January 19, 2015; Accepted March 27, 2015

DOI: $10.3892 / \mathrm{mco} .2015 .546$

\begin{abstract}
The aim of this study was to evaluate whether the addition of rituximab to chemotherapy reduces central nervous system (CNS) events and to identify the risk factors associated with CNS involvement. Patients who were diagnosed with diffuse large B-cell lymphoma (DLBCL) between January, 1995 and December, 2012, without prior CNS disease, were recruited in this study. The patients received chemotherapy with cyclophosphamide, doxorubicin, vincristine and prednisolone (CHOP) or CHOP with rituximab (R-CHOP), with curative intent. The incidence rate of subsequent CNS events was compared between the two groups. A total of 110 patients were recruited, 45 (41\%) of whom received CHOP and 65 (59\%) R-CHOP. A total of 12 patients (10.9\%) subsequently exhibited CNS involvement. The median time from the initial DLBCL diagnosis to CNS disease was 6.7 months (range, 1.3-23.8 months). The CNS disease rate was $15.5 \%(7 / 45)$ in the CHOP group vs. $7.6 \%$ (5/65) in the R-CHOP group. The projected 3-year CNS disease rate was $18 \%$ in the $\mathrm{CHOP}$ group vs. $9 \%$ in the $\mathrm{R}-\mathrm{CHOP}$ group $(\mathrm{P}=0.15)$. The survival of patients with CNS disease was poor, with a median survival of 5.8 months. On multivariate analysis using the Cox proportional model, stage IV disease remained an independent predictor of CNS disease (hazard ratio $=7.75$, 95\% confidence interval: $1.67-35.92, \mathrm{P}=0.009)$. In conclusion, the addition of rituximab to chemotherapy did not appear to reduce the risk of CNS events in our study. Other effective prophylactic measures are required to reduce the incidence of
\end{abstract}

Correspondence to: Dr Man Fai Law, Department of Medicine and Therapeutics, Prince of Wales Hospital, 30-32 Ngan Shing Street, Sha Tin, Hong Kong SAR 999077, P.R. China

E-mail: mflaw99@yahoo.com.hk

*Contributed equally

Key words: rituximab, central nervous system events, diffuse large B-cell lymphoma
CNS events. High-dose intravenous methotrexate crosses the blood-brain barrier and may be used as CNS prophylaxis in high-risk patients.

\section{Introduction}

Diffuse large B-cell lymphoma (DLBCL) is the most common type of non-Hodgkin's lymphoma (1). DLBCL is responsive to chemotherapy and patients with relapsed or refractory disease may be treated with salvage chemotherapy followed by autologous stem cell transplantation (ASCT). In a previous study, the 3 -year overall survival rate was $49 \%$ for relapsed lymphoma without central nervous system (CNS) involvement (2). Patients with disease progression into the CNS exhibit poor survival, despite aggressive interventions, with a median survival of 2-5 months (3-5). The reported incidence of CNS involvement varies from 2.8 to $25 \%$, depending on the population under investigation and the diagnostic tests used (5-7). The CNS lymphoma may involve the brain parenchyma and/or the leptomeninges. CNS may be the only disease site or may be associated with other sites of disease at relapse. There is currently no consensus regarding the optimal strategy to prevent CNS dissemination. Traditionally, the prophylactic measures include intrathecal (IT) injection of methotrexate, cytarabine and steroid, mainly in high-risk patients, i.e., those with marrow, testicular, orbital and nasal sinus involvement.

The addition of rituximab to cyclophosphamide, doxorubicin, vincristine and prednisolone (CHOP) chemotherapy has improved the remission rate and the overall survival in DLBCL patients. Considering the effective eradication of systemic disease in responsive patients, we were prompted to investigate whether rituximab was also able to reduce CNS events. This is a major practical question, since CNS prophylactic measures may require improvement if $\mathrm{CHOP}$ with the addition of rituximab (R-CHOP) is found to be inadequate. The aim of this study was to perform a historical analysis of the patients treated with CHOP and R-CHOP to evaluate whether the CNS disease rate was reduced with the addition of rituximab and to identify the risk factors associated with CNS involvement. 


\section{Patients and methods}

Patient selection and treatment. Patients aged $\geq 18$ years, diagnosed with DLBCL and treated with CHOP or R-CHOP chemotherapy with curative intent in the Tuen Mun Hospital (Hong Kong, China) between January, 1996 and December, 2012, were recruited in this study. The exclusion criteria included human immunodeficiency virus (HIV) positivity and CNS disease at initial diagnosis.

For early-stage patients (stage I or II) with non-bulky disease, 4 courses of CHOP (cyclophosphamide $750 \mathrm{mg} / \mathrm{m}^{2}$ on day 1 , doxorubicin $50 \mathrm{mg} / \mathrm{m}^{2}$ on day 1 , vincristine $1.4 \mathrm{mg} / \mathrm{m}^{2}$ on day 1 and prednisolone $40 \mathrm{mg} / \mathrm{m}^{2}$ on days 1-5) or R-CHOP (addition of rituximab $375 \mathrm{mg} / \mathrm{m}^{2}$ on day 1) were administered. Following chemotherapy, regional radiation therapy (RT) was delivered to the involved area. For patients with advanced stages (III or IV) or early-stage bulky disease, 6-8 courses of CHOP or R-CHOP were administered. The chemotherapy or chemoimmunotherapy were administered every 3 weeks. Patients with bulky disease also received RT. Bulky disease was defined as a mediastinal mass with a maximal width exceeding one-third of the maximal diameter on a standing posteroanterior chest X-ray, or any mass $>10 \mathrm{~cm}$ in diameter. IT prophylaxis with 8 doses of methotrexate $(12 \mathrm{mg})$ was administered to high-risk patients, such as those with bone marrow, testicular, nasal sinus, kidney or breast involvement, unless patients declined prophylactic treatment.

The evaluation of CNS involvement was performed as clinically indicated and included computed tomography (CT) or magnetic resonance imaging (MRI) and lumbar puncture with cerebrospinal fluid (CSF) analysis by cytological examination. CSF analysis was also performed in high-risk patients, such as those with bone marrow, nasal sinus or testicular involvement. CNS involvement was defined as the presence of lymphoma cells in the CSF or the presence of typical lesion(s) on CT or MRI. The rate of CNS disease progression was compared between the CHOP and the R-CHOP groups.

Endpoints. Baseline parameters such as age, disease stage, marrow disease, bulky disease, B symptoms, number and sites of extranodal disease, international prognostic index (IPI) score and lactate dehydrogenase (LDH) level were collected. The prognostic factors associated with subsequent CNS disease were investigated. The primary endpoint was the time-to-CNS disease, which was calculated from the date of pathological diagnosis of lymphoma to the date of diagnosis of CNS disease. Patients not developing CNS lymphoma were censored at the last date of follow-up.

Statistical analysis. Time-to-CNS disease and survival were compared between the two groups using the Kaplan-Meier method and the log-rank test. Baseline parameters associated with CNS disease were compared using the Chi-square test and the Fisher's exact test was used for categorical variables. To evaluate the risk factors for CNS events, a univariate analysis was initially performed using time-to-CNS disease as the endpoint. Subsequently, the Cox proportional hazards model was applied in the multivariate analysis to include factors with $\mathrm{P}<0.05$ in the univariate analysis and assess the effect of these factors on the risk of CNS events. Overall survival was calculated from the date of DLBCL diagnosis until the date of death or censored at the date of the last follow-up. All the $\mathrm{P}$-values were two-sided and $\mathrm{P}<0.05$ was considered to indicate statistically significant differences. Data were analyzed using SPSS software, version 11 (SPSS Inc., Chicago, IL, USA).

\section{Results}

Baseline characteristics. A total of 111 patients with DLBCL were identified and 1 patient who was HIV-positive was excluded from the study. The final cohort included 110 patients, with 45 (41\%) receiving CHOP and 65 (59\%) receiving $\mathrm{R}-\mathrm{CHOP}$. The baseline parameters were comparable between the two groups. Over $90 \%$ of the patients in this study received planned doses of chemotherapy. The median patient age was 55 years (range, 20-77 years) for the CHOP group and 56 years (range, 18-86 years) for the R-CHOP group. The majority of the patients had advanced stage III or IV disease (69\% in the CHOP group and $74 \%$ in the R-CHOP group). A total of $20 \%$ of all the patients exhibited marrow involvement and $21 \%$ exhibited involvement of $>1$ extranodal sites. The extranodal sites included bone marrow, breast, kidney, liver, ovary, nasopharynx and lungs (Table I).

The compliance of patients to IT prophylaxis was low, with a total of $5(11.1 \%)$ and $9(13.8 \%)$ patients in the CHOP and $\mathrm{R}$-CHOP groups, respectively, receiving IT prophylaxis.

Characteristics of CNS diseases. The median follow-up time for patients treated with CHOP and R-CHOP was 58 months (range, 1.1-207 months) and 32 months (range, 1.2-131 months), respectively. In agreement with previously published studies, patients treated with R-CHOP exhibited a higher complete remission rate compared with those treated with $\mathrm{CHOP}$ ( 69 vs. $40 \%$, respectively; $\mathrm{P}=0.003$ ) and a higher 5 -year overall survival (70 vs. $49 \%$, respectively; $\mathrm{P}=0.01$ ) (data not shown).

The median time from diagnosis to CNS disease was 6.7 months (range, 1.3-23.8 months). A total of 4 patients developed CNS lymphoma following initial complete remission (1 patient received CHOP chemotherapy and relapsed 8 months after initial remission; the remaining 3 patients received R-CHOP therapy and relapsed 5-14 months after their initial remission).

A total of 12 patients (10.9\%) subsequently developed CNS involvement; 3 patients received IT prophylaxis and 4 patients exhibited involvement of $>1$ extranodal site. The incidence of CNS events was $15.5 \%$ (7/45) in patients receiving CHOP and $7.6 \%(5 / 65)$ in those receiving $\mathrm{R}-\mathrm{CHOP}$. The projected risk of CNS events at 3 years was 9\% in the R-CHOP group compared with $18 \%$ in the CHOP group $(\mathrm{P}=0.15)$ (Fig. 1). Parenchymal relapse appeared to be more common among patients receiving R-CHOP (R-CHOP vs. CHOP, 80 vs. $29 \%$, respectively) (Table II).

Treatment of CNS disease. A total of 8 patients suffering from CNS disease were re-treated with high-dose methotrexate and IT methotrexate and cytarabine; 5 of these patients also underwent whole-brain RT; 2 patients with systemic and CNS disease were administered dexamethasone, cytarabine and cisplatin chemotherapy, IT methotrexate and cytarabine; and 2 patients received supportive therapy due to their advanced 
Table I. Baseline characteristics of the two treatment groups.

\begin{tabular}{|c|c|c|c|}
\hline Characteristics & $\begin{array}{c}\mathrm{CHOP}^{\mathrm{a}}, \text { no. }(\%) \\
(\mathrm{n}=45)\end{array}$ & $\begin{array}{c}\mathrm{R}-\mathrm{CHOP}^{\mathrm{b}}, \text { no. }(\%) \\
(\mathrm{n}=65)\end{array}$ & P-value \\
\hline \multicolumn{4}{|l|}{ Age, years } \\
\hline$<60$ & $31(69.0)$ & $41(63.0)$ & 0.55 \\
\hline$\geq 60$ & $14(31.0)$ & $24(37.0)$ & 0.55 \\
\hline \multicolumn{4}{|l|}{ Gender } \\
\hline Male & $21(47.0)$ & $27(42.0)$ & 0.69 \\
\hline Female & $24(53.0)$ & $38(58.0)$ & 0.69 \\
\hline $\mathrm{LDH}>2 \mathrm{xULN}$ & $17(39.0)$ & $23(35.0)$ & 0.84 \\
\hline \multicolumn{4}{|l|}{ Stage } \\
\hline III or IV & $31(69.0)$ & $48(74.0)$ & 0.67 \\
\hline IV & $17(38.0)$ & $31(47.0)$ & 0.33 \\
\hline B symptoms & $23(51.0)$ & $39(60.0)$ & 0.43 \\
\hline Bulky disease & $2(4.4)$ & $8(12.0)$ & 0.19 \\
\hline ECOG performance status $>1$ & $3(6.6)$ & $7(11.0)$ & 0.52 \\
\hline IPI 3-5 & $15(33.0)$ & $12(48.0)$ & 0.17 \\
\hline Extranodal sites $>1$ & $6(13.0)$ & $17(26.0)$ & 0.15 \\
\hline Bone marrow involvement & $10(22.0)$ & $12(18.0)$ & 0.63 \\
\hline Breast involvement & $2(4.5)$ & $1(1.5)$ & 0.56 \\
\hline Nasopharyngeal involvement & $1(2.2)$ & $4(6.1)$ & 0.65 \\
\hline Kidney involvement & $1(2.2)$ & $2(3.1)$ & 1.00 \\
\hline Pulmonary involvement & $4(8.9)$ & $10(15.3)$ & 0.39 \\
\hline Hepatic involvement & $1(2.2)$ & $2(3.1)$ & 1.00 \\
\hline Ovarian involvement & $1(2.2)$ & $2(3.1)$ & 1.00 \\
\hline CNS prophylaxis with IT chemotherapy & $5(11.1)$ & $9(13.8)$ & 0.77 \\
\hline
\end{tabular}

${ }^{a}$ Cyclophosphamide, doxorubicin, vincristine and prednisolone. ${ }^{\mathrm{b}} \mathrm{CHOP}$ with rituximab. LDH, lactate dehydrogenase; ULN, upper limit of normal; ECOG, Eastern Cooperative Oncology Group; IPI, international prognostic index; CNS, central nervous system; IT, intrathecal.

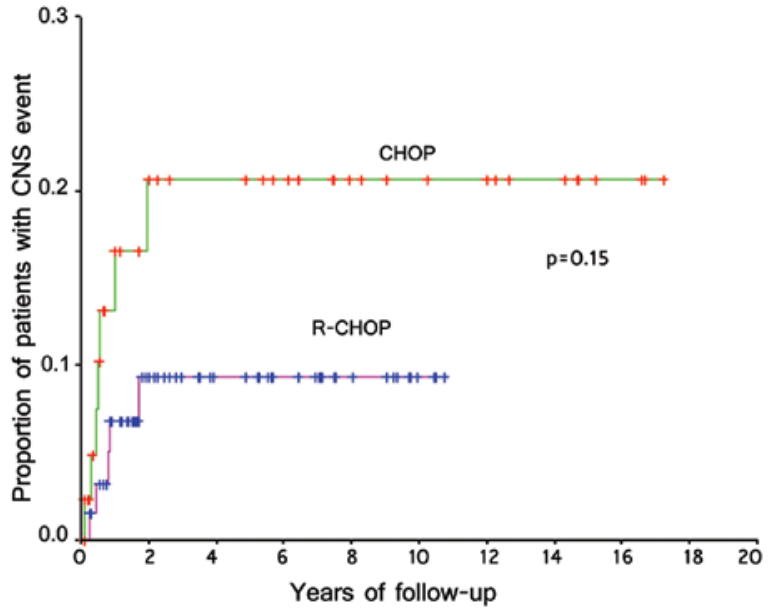

Figure 1. Time-to-central nervous system (CNS) event in CHOP- and R-CHOP-treated patients.

age and refractory lymphoma. However, the survival of all patients with CNS disease was poor, with a median survival time of 5.8 months.
Risk factors for CNS involvement. On univariate analysis, the following factors were significantly associated with subsequent secondary CNS disease: Stage IV disease [hazard ratio $(\mathrm{HR})=8.79,95 \%$ confidence interval $(\mathrm{CI}): 1.91-40.34, \mathrm{P}=0.005]$, bone marrow involvement $(\mathrm{HR}=5.24,95 \% \mathrm{CI}: 1.69-16.31)$, $\mathrm{P}=0.004)$ and elevated LDH level $>2$ times the upper limit of normal (HR=3.01, 95\% CI: 0.97-9.61, $\mathrm{P}=0.05$ ) (Table III). On multivariate analysis using the Cox proportional model, stage IV disease remained an independent predictor for CNS disease $(\mathrm{HR}=7.75,95 \% \mathrm{CI}: 1.67-35.92, \mathrm{P}=0.009)$ (data not shown).

\section{Discussion}

The addition of rituximab to $\mathrm{CHOP}$ has been shown to improve the remission rate and the overall and event-free survival of patients with DLBCL (8-11). Based on the eradication of systemic disease achieved by rituximab, we investigated whether it was able to reduce the incidence of CNS involvement since its introduction into our practice. The overall CNS event rate for DLBCL was $10.9 \%$ in our study. The majority of CNS events developed within 1 year of the diagnosis of 
Table II. Characteristics of patients with central nervous system (CNS) relapse.

\begin{tabular}{|c|c|c|}
\hline Characteristics & $\begin{array}{c}\mathrm{CHOP}^{\mathrm{a}} \text {, no. }(\%) \\
(\mathrm{n}=7)\end{array}$ & $\begin{array}{c}\mathrm{R}^{-\mathrm{CHOP}^{\mathrm{b}}}, \text { no. }(\%) \\
(\mathrm{n}=5)\end{array}$ \\
\hline \multicolumn{3}{|l|}{ Site of CNS relapse } \\
\hline Parenchymal & $2(29.0)$ & $4(80.0)$ \\
\hline Leptomeningeal & $5(71.0)$ & $1(20.0)$ \\
\hline \multicolumn{3}{|l|}{ Diagnosis of CNS relapse } \\
\hline Imaging & $2(29.0)$ & $4(80.0)$ \\
\hline CSF & $3(42.0)$ & $1(20.0)$ \\
\hline Both & $2(29.0)$ & $0(0.0)$ \\
\hline Relapse within the first year from diagnosis & $5(71.0)$ & $4(80.0)$ \\
\hline \multicolumn{3}{|l|}{ Treatment of CNS relapse } \\
\hline High-dose methotrexate & $5(71.0)$ & $3(60.0)$ \\
\hline IT chemotherapy & $6(86.0)$ & $4(80.0)$ \\
\hline Whole-brain irradiation & $2(29.0)$ & $3(60.0)$ \\
\hline Supportive therapy & $1(14.0)$ & $1(20.0)$ \\
\hline \multicolumn{3}{|l|}{ Outcome } \\
\hline Lymphoma-related death & $6(86.0)$ & $4(80.0)$ \\
\hline Alive in second remission & $1(14.0)$ & $1(20.0)$ \\
\hline
\end{tabular}

${ }^{a}$ Cyclophosphamide, doxorubicin, vincristine and prednisolone. ${ }^{\mathrm{b}} \mathrm{CHOP}$ with rituximab. CSF, cerebrospinal fluid; IT, intrathecal.

Table III. Risk factors for central nervous system relapse in univariate analysis.

\begin{tabular}{llc}
\hline Factors & Hazard ratio $(95 \%$ CI $)$ & P-value \\
\hline Age $>60$ years & $0.39(0.09-1.78)$ & 0.23 \\
Male gender & $2.01(0.63-6.33)$ & 0.23 \\
LDH $>1 x$ ULN & $1.21(0.26-5.51)$ & 0.81 \\
LDH $>2 x$ ULN & $3.01(0.97-9.61)$ & 0.05 \\
Bone marrow involvement & $5.24(1.69-16.31)$ & 0.004 \\
Stage IV disease & $8.79(1.91-40.34)$ & 0.005 \\
Stage III and IV disease & $5.27(0.68-40.9)$ & 0.11 \\
Extranodal sites $>1$ & $2.39(0.71-8.03)$ & 0.15 \\
IPI $>2$ & $1.12(0.35-3.54)$ & 0.84 \\
B symptoms & $1.69(0.51-5.61)$ & 0.39 \\
Bulky disease & $2.43(0.53-11.15)$ & 0.25 \\
IT prophylaxis & $2.69(0.74-10.0)$ & 0.14 \\
Rituximab & $0.43(0.14-1.38)$ & 0.15 \\
\hline
\end{tabular}

CI, confidence interval; LDH, lactate dehydrogenase; ULN, upper limit of normal; IPI, international prognostic index; IT, intrathecal.

lymphoma. The CNS disease rate was $15.5 \%(7 / 45)$ in the CHOP group vs. $7.6 \%(5 / 65)$ in the R-CHOP group. The projected 3-year CNS disease rate was $18 \%$ in the $\mathrm{CHOP}$ group vs. $9 \%$ in the $\mathrm{R}-\mathrm{CHOP}$ group $(\mathrm{P}=0.15)$. Therefore, the addition of rituximab to chemotherapy did not significantly reduce the risk of CNS events in our study.

The risk of CNS events was higher in our cohort compared with that in previous studies. The CNS disease rate was previously reported to be $2.2-10.4 \%(3,5,12-15)$. This difference may be attributed to our inclusion of more patients with advanced-stage (III or IV) disease $(\sim 70 \%)$. Moreover, lumbar puncture with CSF analysis was not routinely performed at diagnosis to allow for the detection and exclusion of patients with occult CNS disease. The early CNS relapse and higher proportion of isolated CNS relapses observed in our study may also reflect the presence of subclinical CNS disease at diagnosis.

Stage IV lymphoma was identified as a significant predictor of CNS disease in our cohort. Elevated LDH levels, extranodal site involvement and advanced stage of lymphoma are the most 
commonly reported risk factors. Our findings are consistent with those of previous studies (12-17).

Rituximab penetrates poorly across the blood-brain barrier. A pharmacokinetic study demonstrated that, following an intravenous (i.v.) dose of rituximab, its levels in the CSF were only $0.1 \%$ of their corresponding levels in the serum (18). Therefore, it is unlikely that rituximab is able to directly access the lymphoma cells in the CNS. We observed a relatively higher proportion of parenchymal disease in the R-CHOP group compared with that in the CHOP group (80 vs. $29 \%$, respectively). The pharmacokinetic constraints of rituximab may limit its ability to prevent lymphoma progression in the parenchymal compartment. We also observed that isolated CNS disease was more common in the R-CHOP group in our cohort. This may be due to the fact that R-CHOP is more effective in preventing systemic relapse of lymphoma.

The optimal treatment for patients with disease progression into the CNS has not yet been standardized $(19,20)$. The majority of our patients were treated with high-dose methotrexate, with or without whole-brain RT. It was previously demonstrated that treatment with high-dose methotrexate was effective and improved survival (19). Whole-brain RT is effective for initial control of CNS lymphoma; however, it is associated with increased risk of delayed treatment-related neurotoxicity, particularly in elderly patients (21). There is also a potential role for high-dose chemotherapy followed by ASCT. Bromberg et al reported an overall survival benefit in patients undergoing ASCT and long-term survival is more likely in these patients (22).

Our study was limited by its retrospective nature and the small sample size. Not all patients underwent lumbar puncture and CSF analysis at the beginning of therapy; therefore, a proportion of patients with subclinical CNS disease at diagnosis may have been missed. There was also a difference in follow-up time between the two groups.

The efficacy of IT prophylaxis could not be properly assessed in our study due to the low compliance. Previous published studies on the effect of rituximab on CNS events also reported a similar low rate of IT prophylaxis, even in high-risk patients $(4,13,14)$. It was found the effect of rituximab on the risk of CNS disease could be assessed regardless of whether IT prophylaxis had been administered (4). Although certain studies support the efficacy of IT chemotherapy, several others have questioned its ability to prevent CNS recurrence (14,23-26). Although IT chemotherapy has been effective in preventing or treating leptomeningeal disease, its efficacy in preventing parenchymal disease has been questioned due to the low penetration into the brain parenchyma and the uneven distribution within the neuroaxis. Lumbar administration of IT methotrexate also results in marked differences in peak levels throughout the subarachnoid space. Subtherapeutic levels are common due to differences in CSF movement, choroidal uptake and drug clearance (27). IT prophylaxis is associated with several rare but severe neurological complications, such as seizures, encephalopathy and spinal cord lesions manifesting as tetraplegia, paraplegia and cauda equina syndrome (28). These complications may defer patients receiving IT chemotherapy.

Patients with CNS relapse have a poor prognosis. The incidence of CNS events may be reduced by increasing the sensitivity of diagnosis of CNS disease and applying more effective prophylactic therapeutic regimens. The application of more sensitive tests may facilitate the diagnosis of occult CNS disease. Flow cytometry of CSF may prove useful for the detection of leptomeningeal involvement and it is more sensitive compared with conventional cytological analysis of CSF (29). Brain MRI or CT scan is indicated for the detection of parenchymal involvement, particularly in high-risk patients, such as those with stage IV DLBCL. More intensive upfront CNS-directed prophylaxis may be used for high-risk patients. High-dose i.v. methotrexate or cytosine arabinoside may cross the blood-brain barrier and have been used to treat established CNS disease. The incorporation of high-dose i.v. methotrexate into the rituximab combination may be a rational prophylactic approach for high-risk patients. A retrospective study with a median of 3 cycles of i.v. methotrexate $3.5 \mathrm{~g} / \mathrm{m}^{2}$ administered to a high-risk group of DLBCL patients reported a significant reduction of CNS recurrence, with a recurrence rate of only $3 \%$ in the high-risk group at a median follow-up of 33 months (30).

Another multicenter retrospective study of patients at high-risk for CNS relapse demonstrated that the addition of high-dose i.v. methotrexate and/or cytarabine was associated with a lower incidence of CNS relapse compared with IT chemotherapy alone (31). The 3-year actuarial rates of CNS relapse in the groups receiving IT methotrexate, IT methotrexate with 2 cycles of high-dose i.v. methotrexate, and chemotherapy regimens containing high-dose i.v. methotrexate and cytarabine, were $18.4,6.9$ and $2.3 \%$, respectively $(\mathrm{P}=0.009)$. The most frequent toxicity of i.v. methotrexate was renal impairment, which was grade 1 in the majority of the cases.

A prospective study of patients with DLBCL or grade III follicular lymphoma reported that 6 courses of R-CHEOP (rituximab, cyclophosphamide, doxorubicin, etoposide, vincristine and prednisolone) followed by high-dose cytarabine ( $3 \mathrm{~g} / \mathrm{m}^{2}$ twice daily for 2 days) and a course of high-dose methotrexate ( $3 \mathrm{~g} / \mathrm{m}^{2}$ i.v. as a $24-\mathrm{h}$ infusion) achieved a CNS relapse rate of $4.5 \%$ (32). There is growing evidence that high-dose antimetabolite therapy may provide effective CNS prophylaxis in patients with DLBCL.

The optimal time of systemic high-dose chemotherapy has not yet been defined. CNS relapse is most common within the first 12 months from the completion of primary therapy $(14,25-26,33)$. This may suggest the presence of occult CNS disease at diagnosis and early use of high-dose i.v. methotrexate is recommended.

In conclusion, although the addition of rituximab to chemotherapy may improve the remission rate and overall survival of patients with DLBCL, it did not appear to decrease the risk of CNS events in our study. There is a need for a better prophylactic strategy by increasing the sensitivity of diagnosis of CNS disease and more effective prophylactic therapeutic regimens, such as high-dose i.v. methotrexate, in order to reduce CNS events, particularly in high-risk patients.

\section{References}

1. Martelli M, Ferreri AJ, Agostinelli C, Di Rocco A, Pfreundschuh M and Pileri SA: Diffuse large B-cell lymphoma. Crit Rev Oncol Hematol 87: 146-171, 2013.

2. Gisselbrecht C, Glass B, Mounier N, et al: Salvage regimens with autologous transplantation for relapsed large B-cell lymphoma in the rituximab era. J Clin Oncol 28: 4184-4190, 2010. 
3. Feugier P, Virion JM, Tilly H, Haioun C, Marit G, Macro M, Bordessoule D, Recher C, Blanc M, Molina T, Lederlin P and Coiffier B: Incidence and risk factors for central nervous system occurrence in elderly patients with diffuse large-B-cell lymphoma: influence of rituximab. Ann Oncol 15: 129-133, 2004.

4. Boehme V, Schmitz N, Zeynalova S, Loeffler M and Pfreundschuh M: CNS events in elderly patients with aggressive lymphoma treated with modern chemotherapy (CHOP-14) with or without rituximab: an analysis of patients treated in the RICOVER-60 trial of the German High-Grade Non-Hodgkin Lymphoma Study Group (DSHNHL). Blood 113: 3896-3902, 2009.

5. Bernstein SH, Unger JM, Leblanc M, Friedberg J, Miller TP and Fisher RI: Natural history of CNS relapse in patients with aggressive non-Hodgkin's lymphoma: a 20 -year follow-up analysis of SWOG 8516-the Southwest Oncology Group. J Clin Oncol 27: 114-119, 2009.

6. Bierman P and Giglio P: Diagnosis and treatment of central nervous system involvement in non-Hodgkin's lymphoma. Hematol Oncol Clin North Am 19: 597-609, 2005.

7. Hegde U, Filie A, Little RF, Janik JE, Grant N, Steinberg SM, Dunleavy K, Jaffe ES, Abati A, Stetler-Stevenson M and Wilson WH: High incidence of occult leptomeningeal disease detected by flow cytometry in newly diagnosed aggressive B-cell lymphomas at risk for central nervous system involvement: the role of flow cytometry vs. cytology. Blood 105: 496-502, 2005.

8. Coiffier B, Lepage E, Briere J, et al: CHOP chemotherapy plus rituximab compared with CHOP alone in elderly patients with diffuse large-B-cell lymphoma. N Engl J Med 346: 235-242, 2002.

9. Coiffier B, Thieblemont C, Van Den Neste E, et al: Long-term outcome of patients in the LNH-98.5 trial, the first randomized study comparing rituximab-CHOP to standard CHOP chemotherapy in DLBCL patients: a study by the Groupe d'Etudes des Lymphomes de l'Adulte. Blood 116: 2040-2045, 2010.

10. Pfreundschuh M, Trümper L, Osterborg A, et al; MabThera International Trial Group: CHOP-like chemotherapy plus rituximab vs. CHOP-like chemotherapy alone in young patients with good prognosis diffuse large-B-cell lymphoma: a randomised controlled trial by the MabThera International Trial (MInT) Group. Lancet Oncol 7: 379-391, 2006.

11. Pfreundschuh M,Schubert J,Ziepert M, et al; German High-Grade Non-Hodgkin Lymphoma Study Group (DSHNHL): Six versus eight cycles of bi-weekly CHOP-14 with or without rituximab in elderly patients with aggressive $\mathrm{CD} 20^{+} \mathrm{B}$-cell lymphomas: a randomized controlled trial (RICOVER-60). Lancet Oncol 9: 105-116, 2008.

12. Boehme V, Zeynalova S, Kloess M, Loeffler M, Kaiser U, Pfreundschuh $M$ and Schmitz N; German High-Grade Non-Hodgkin's Lymphoma Study Group (DSHNHL): Incidence and risk factors of central nervous system recurrence in aggressive lymphoma - a survey of 1693 patients treated in protocols of the German High-Grade Non-Hodgkin's Lymphoma Study Group (DSHNHL). Ann Oncol 18: 149-157, 2007.

13. Shimazu Y, Notohara K and Ueda Y: Diffuse large B-cell lymphoma with central nervous system relapse: prognosis and risk factors according to retrospective analysis from a single-center experience. Int J Hematol 89: 577-583, 2009.

14. Villa D, Connors JM, Shenkier TN, Gascoyne RD, Sehn LH and Savage KJ: Incidence and risk factors for central nervous system relapse in patients with diffuse large B-cell lymphoma: the impact of the addition of rituximab to CHOP chemotherapy. Ann Oncol 21: 1046-1052, 2010.

15. Kridel R and Dietrich PY: Prevention of CNS relapse in diffuse large B-cell lymphoma. Lancet Oncol 12: 1258-1266, 2011.

16. Jahnke K, Thiel E, Martus P, Schwartz S and Korfel A: Retrospective study of prognostic factors in non-Hodgkin lymphoma secondarily involving the central nervous system. Ânn Hematol 85: 45-50, 2006.

17. Tomita N, Yokoyama M, Yamamoto W, et al: Central nervous system event in patients with diffuse large B-cell lymphoma in rituximab era. Cancer Sci 103: 245-251, 2012.

18. Rubenstein JL, Combs D, Rosenberg J, Levy A, McDermott M, Damon L, Ignoffo R, Aldape K, Shen A, Lee D, Grillo-Lopez A and Shuman MA: Rituximab therapy for CNS lymphomas: targeting the leptomeningeal compartment. Blood 101: 466-468, 2003
19. Doolittle ND, Abrey LE, Shenkier TN, et al: Brain parenchyma involvement as isolated central nervous system relapse of systemic non-Hodgkin lymphoma: an International Primary CNS Lymphoma Collaborative Group report. Blood 111: 1085-1093, 2008

20. Kim SJ, Oh SY, Kim JS, Kim H, Lee GW, Won JH, Shin HJ, Yang DH, Choi CW, Park J, Kim WS and Suh C: Secondary central nervous system (CNS) involvement in patients with diffuse large B-cell lymphoma: a therapeutic dilemma. Ann Hematol 90: 539-546, 2011.

21. Gavrilovic IT, Hormigo A, Yahalom J, DeAngelis LM and Abrey LE: Long-term follow-up of high-dose methotrexate-based therapy with and without whole brain irradiation for newly diagnosed primary CNS lymphoma. J Clin Oncol 24: 4570-4574, 2006.

22. Bromberg JE, Doorduijn JK, Illerhaus G, Jahnke K, Korfel A Fischer L, Fritsch K, Kuittinen O, Issa S, van Montfort C and van den Bent MJ: Central nervous system recurrence of systemic lymphoma in the era of stem cell transplantation - an International Primary Central Nervous System Lymphoma Study Group project. Haematologica 98: 808-813, 2013.

23. Chua SL, Seymour JF, Streater J, Wolf MM, Januszewicz EH and Prince HM: Intrathecal chemotherapy alone is inadequate central nervous system prophylaxis in patients with intermediate-grade non-Hodgkin's lymphoma. Leuk Lymphoma 43: 1783-1788, 2002.

24. Arkenau HT, Chong G, Cunningham D, Watkins D, Agarwal R, Sirohi B, Trumper M, Norman A, Wotherspoon A and Horwich A: The role of intrathecal chemotherapy prophylaxis in patients with diffuse large B-cell lymphoma. Ann Oncol 18: 541-545, 2007.

25. Tai WM, Chung J, Tang PL, Koo YX, Hou X, Tay KW, Quek R, Tao M and Lim ST: Central nervous system (CNS) relapse in diffuse large B cell lymphoma (DLBCL): pre- and post-rituximab. Ann Hematol 90: 809-818, 2011.

26. Schmitz N, Zeynalova S, Glass B, Kaiser U, Cavallin-Stahl E, Wolf M, Haenel M, Loeffler M, Truemper L and Pfreundschuh M: CNS disease in younger patients with aggressive B-cell lymphoma: an analysis of patients treated on the Mabthera international trial and trials of the German High-Grade Non-Hodgkin Lymphoma Study Group. Ann Oncol 23: 1267-1273, 2012.

27. Shapiro WR, Young DF and Mehta BM: Methotrexate: distribution in cerebrospinal fluid after intravenous, ventricular and lumbar injections. N Engl J Med 293: 161-166, 1975.

28. Kwong YL, Yeung DY and Chan JC: Intrathecal chemotherapy for hematologic malignancies: drugs and toxicities. Ann Hematol 88: 193-201, 2009.

29. Hegde U, Filie A, Little RF, Janik JE, Grant N and Steinberg SM: High incidence of occult leptomeningeal disease detected by flow cytometry in newly diagnosed aggressive B-cell lymphomas at risk for CNS involvement: the role of flow cytometry vs. cytology. Blood 105: 496-502, 2005.

30. Abramson JS, Hellmann M, Barnes JA, Hammerman P, Toomey C, Takvorian T, Muzikansky A and Hochberg EP: Intravenous methotrexate as central nervous system (CNS) prophylaxis is associated with a low risk of CNS recurrence in high-risk patients with diffuse large B-cell lymphoma. Cancer 116: 4283-4290, 2010

31. Cheah CY, Herbert KE, O'Rourke K, et al: A multicentre retrospective comparison of central nervous system prophylaxis strategies among patients with high-risk diffuse large B-cell lymphoma. Br J Cancer 111: 1072-1079, 2014.

32. Holte H, Leppä S, Björkholm M, et al: Dose-densified chemoimmunotherapy followed by systemic central nervous system prophylaxis for younger high-risk diffuse large B-cell/follicular grade 3 lymphoma patients: results of a phase II Nordic Lymphoma Group study. Ann Oncol 24: 1385-1392, 2013.

33. Yamamoto W, Tomita N, Watanabe R, Hattori Y, Nakajima Y, Hyo R, Hashimoto C, Motomura S and Ishigatsubo Y: Central nervous system involvement in diffuse large B-cell lymphoma. Eur J Haematol 85: 6-10, 2010. 